\title{
Frondoside A from sea cucumber and nymphaeols from Okinawa propolis: Natural anti-cancer agents that selectively inhibit PAK1 in vitro
}

\author{
Binh Cao Quan Nguyen ${ }^{1}$, Kazuki Yoshimura ${ }^{2}$, Shigenori Kumazawa ${ }^{2}$, Shinkichi Tawata ${ }^{1}$, \\ Hiroshi Maruta,* \\ ${ }^{1}$ PAK Research Center, University of the Ryukyus, Okinawa, Japan; \\ ${ }^{2}$ Department of Food and Nutritional Sciences, University of Shizuoka, Shizuoka, Japan; \\ ${ }^{3}$ PAK Research Center, Melbourne, Australia.
}

\begin{abstract}
Summary A sulfated saponin called "Frondoside A" (FRA) from sea cucumber and ingredients from Okinawa propolis (OP) have been previously shown to suppress the PAK1-dependent growth of A549 lung cancer as well as pancreatic cancer cells. However, the precise molecular mechanism underlying their anti-cancer action still remains to be clarified. In this study, for the first time, we found that both FRA and OP directly inhibit PAK1 in vitro in a selective manner (far more effectively than two other oncogenic kinases, LIMK and AKT). Furthermore, at least two major anti-cancer ingredients of OP, nymphaeols $A$ and $C$, also directly inhibit PAK1 in vitro in a selective manner. To the best of our knowledge, FRA is the first marine compound that selectively inhibits PAK1. Likewise, these nymphaeols are the first propolis ingredients that selectively inhibit PAK1.
\end{abstract}

Keywords: Propolis, sea cucumber, frondoside A, nymphaeols, PAK1, cancers

\section{Introduction}

Since conventional chemotherapeutics such as DNA/ microtubule poisons cause serious side effects such as hair-loss and suppression of immune response, recently cancer patients, in particular those who suffer from formidable pancreatic or lung cancers, started seeking an alternative approach for cancer therapy by using natural remedies that do not cause any serious side effect. A bee product called "propolis" has been used as one of these herbal cancer therapeutics for last three decades. Two major propolis products available in the market are ARC (artepillin C)-based Brazilian green propolis (GP) and CAPE (caffeic acid phenethyl ester)-based propolis called Bio 30 or Bio 100 from New Zealand (13). However, recently, propolis from subtropical regions

Released online in J-STAGE as advance publication April 24, 2017.

*Address correspondence to:

Dr. Hiroshi Maruta, PAK Research Center, Melbourne, Australia 3055, Australia.

E-mail: maruta20420@yahoo.co.jp such as Okinawa, Taiwan, and Hawaii has been studied extensively, mainly because of its unique ingredients such as geranylated flavonoids (nymphaeols) (4-6). Very recently, we found that Okinawa propolis (OP) is highly anti-angiogenic in ovo (fertilized eggs), clearly several times more potent than GP as a herbal anti-cancer remedy, and blocks the oncogenic/ageing kinase PAK1 at least in cell culture $(4,7)$. Furthermore, we confirmed that $\mathrm{OP}$ is a potent elixir extending the healthy lifespan of $\mathrm{C}$. elegans (7).

In addition to these three distinct propolis products, the potent anti-cancer activity of a sulfated saponin called "frondoside A" (FRA) from an edible sea cucumber (Cucumaria frondosa) has recently drawn much attention of pancreatic and lung cancer patients. According to previous studies by Thomas Adrian and others, FRA inhibits the growth of A549 lung cancer and pancreatic cancer cells with $\mathrm{IC}_{50}$ ranging $1-3 \mu \mathrm{M}$ in cell culture, and up-regulates the tumor-suppressor $\mathrm{p} 21$, an inhibitor of CDKs (cyclin-dependent kinases) $(8,9)$. In vivo (xenograft in nude mice) FRA (1 mg/kg/day, i.p.) significantly suppresses the growth of human pancreatic cancer (9). We have shown previously that the expression of p21 gene is suppressed by PAK1 (10). Furthermore, 
since both A549 lung cancer and pancreatic cancer cells carry the oncogenic Ki-RAS mutant, and their growth depends on PAK1, it would not be unreasonable for us to suspect that FRA might block the oncogenic/ageing kinase PAK1 somehow. Here, we have confirmed that both OP and FRA as well as nymphaeols directly inhibit PAK1 in vitro in a selective manner.

\section{Materials and Methods}

\subsection{Chemicals and reagents}

Human lung cancer cell line A549 was purchased from Japanese Collection of Research Bioresources Cell Bank (Osaka, Japan). Alcohol extract of Okinawa propolis (OP) was prepared as previously described (7). Nymphaeols were isolated from OP through HPLC as previously described (4). Frondoside A (FRA) was purchased from Sigma-Aldrich (St. Louis, MO, USA). Both recombinant PAK1 and LIMK were obtained from SignalChem Pharmaceuticals Inc. (Richmond, British Columbia, Canada).

\subsection{Assay for anti-cancer activity in cell culture}

The number of viable cells after treatment with either frondoside A or nymphaeols (see Figure 1 for their chemical structures) was measured by Trypan blue assay in a hemocytometer as described previously (11). Briefly, A549 lung cancer cells $\left(2 \times 10^{5}\right.$ cells/well $)$ were seeded for $24 \mathrm{~h}$, and then treated with various test compounds at the indicated concentrations for $72 \mathrm{~h}$, and the number of viable (unstained) cells were counted after Trypan blue staining.

\subsection{In vitro anti-kinase (PAK1/LIMK/AKT) assay}

The kinase activity of PAK1, LIMK, and AKT was measured in vitro by ADP-Glo kinase assay kit (Promega, Madison, WI, USA), according to the manufacturer's instruction, as previously described (12). Briefly, the recombinant human PAK1 (10 ng) or LIMK (10 ng) per reaction was treated with either OP, FRA or nymphaeols at the indicated concentrations in the presence of ATP, with either myelin basic protein (MBP) for PAK1 assay or cofilin for LIMK assay as their protein substrates, during the $40 \mathrm{~min}$ in vitro kinase reaction. Then the reaction was terminated with the $\mathrm{ADP} \neg$-Glo reagent. In the case of AKT assay, instead of using the recombinant AKT, A549 lung cancer cells were cultured for $48 \mathrm{~h}$, and cell lysates were immuno-precipitated (IP) with anti-AKT IgG in the presence of protein A-agarose beads (11), and the resultant AKT in IP was treated with various test compounds at the indicated concentrations in the presence of ATP and MBP during $40 \mathrm{~min}$ in vitro kinase assay, which was then terminated with the ADP-<smiles>CC(C)=CCC/C(C)=C/Cc1c(O)cc2c(c1O)C(=O)C[C@@H](c1ccc(O)c(O)c1)O2</smiles>

Figure 1. Chemical structures of nymphaeols A-C and frondoside A. (A) nymphaeol A; (B) nymphaeol B; (C) nymphaeol C; (D) frondoside A. 
Glo reagent. To these reaction mixtures was added the kinase detection reagent that converts ADP to ATP which eventually generates a luciferin-luciferase based fluorescence.

\subsection{Statistical analysis}

Data are expressed as mean values with their standard errors. Statistical comparisons were performed by oneway ANOVA. Statistical analysis was conducted using SPSS (release 16.0, Chicago, Illinois) and $p<0.05$ was considered significant.

\section{Results and Discussion}

3.1. Okinawa propolis (OP) and its major ingredients directly inhibit PAK1 in vitro

\subsubsection{Okinawa propolis $(O P)$ directly inhibits PAK1 in vitro}

We have previously found that Okinawa propolis (OP) inhibits the PAK1-dependent growth of A549 lung cancer cells with $\mathrm{IC}_{50}$ around $12 \mu \mathrm{g} / \mathrm{mL}$, while it blocks PAK1 in the same cell culture with the apparent $\mathrm{IC}_{50}$ around $6 \mu \mathrm{g} /$ $\mathrm{mL}$ as judged by "Macaroni-Western" ATP-Glo kinase assay (7). Generally speaking, when a compound blocks PAK1 by inhibiting an upstream activator of PAK1 such as RAC, instead of directly inhibiting PAK1, the apparent anti-PAK $\mathrm{IC}_{50}$ value is usually 3-4 times higher than the anti-cancer $\mathrm{IC}_{50}$ value (11). Since the outcome with OP is clearly opposite, the possibility rose that OP might directly inhibit PAK1. Here we have confirmed this notion. As shown in Figure 2, OP directly inhibited the recombinant PAK1 in vitro with $\mathrm{IC}_{50}$ around $10 \mu \mathrm{g} / \mathrm{mL}$.

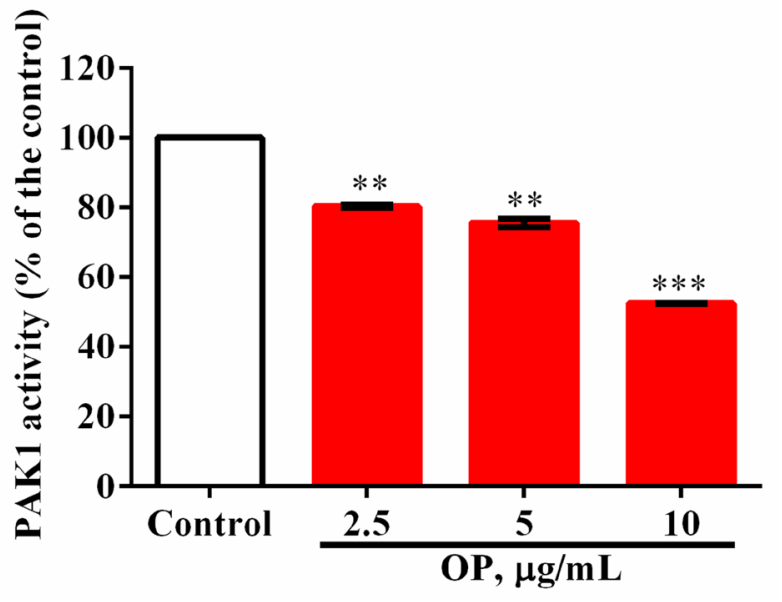

Figure 2. Okinawa propolis (OP) directly inhibits PAK1 in vitro. Recombinant PAK1 from SignalChem was treated with $\mathrm{OP}$ at the indicated concentrations in vitro. The experiments are conducted with twice, and the results are mean $\pm \mathrm{SE} . \mathrm{IC}_{50}$ of OP against PAK1 is around $10 \mu \mathrm{g} / \mathrm{mL}$. Asterisks on each bar indicate significant differences between treatment and control. ${ }^{*} 0.01 \leq p \leq 0.05,{ }^{* *} p \leq 0.01,{ }^{* * *} p \leq 0.001$.

\subsubsection{Anti-cancer activity of nymphaeols $A$ and $C$ from} $O P$

The major anti-cancer ingredients in OP are geranylated flavonoids called nymphaeols A-C (5), and at least nymphaeol A has been shown to inhibit the PAK1dependent angiogenesis in ovo (fertilized eggs) (4), suggesting that it could block PAK1 somehow. Here, we have tested the anti-cancer activity of nymphaeols $\mathrm{A}$ and C. As shown in Figure 3, nymphaeols A and C inhibit the growth of A549 cancer cells with the $\mathrm{IC}_{50}=4$ $\mu \mathrm{M}$ and $7 \mu \mathrm{M}$, respectively.

\subsubsection{Anti-PAK1 activity of nymphaeols $A$ and $C$ in vitro}

The next, we have tested the anti-PAK1 activity in vitro. As shown in Figure 4, like OP, both nymphaeols A and $\mathrm{C}$ directly inhibited PAK1 with $\mathrm{IC}_{50}$ around $10 \mu \mathrm{M}$.

\subsubsection{Kinase specificity of nymphaeols}

In order to determine how selective the direct action of
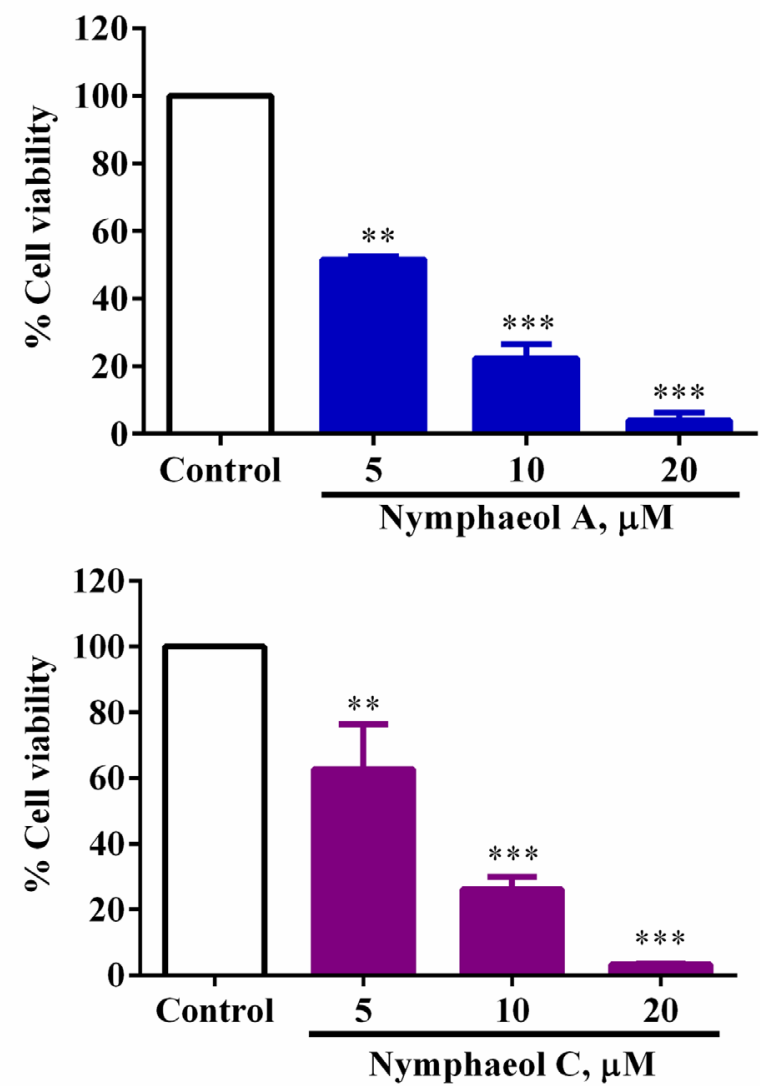

Figure 3. Anti-cancer activity of nymphaeols against the growth of A549 lung cancer cells. A549 cells were treated with either nymphaeols A (left) or C (right) at the indicated concentrations for $72 \mathrm{~h}$, and the number of the viable cells was counted by Trypan blue staining. The results are mean \pm SE of two independent experiments. $\mathrm{IC}_{50}$ of nymphaeols $\mathrm{A}$ and $\mathrm{C}$ are around 4 and $7 \mu \mathrm{M}$, respectively. Asterisks on each bar indicate significant differences between treatment and control. ${ }^{*} 0.01 \leq p \leq 0.05,{ }^{* *} p \leq 0.01,{ }^{* * *} p \leq 0.001$. 
nymphaeols towards PAK1 is, we have tested their antiLIM kinase (LIMK) and anti-AKT activity in vitro as well. As summarized in Table 1, both nymphaeols A and $\mathrm{C}$ inhibited LIMK and AKT, but with the far higher $\mathrm{IC}_{50}(160 \mu \mathrm{M}$ and $170 \mu \mathrm{M}$ against LIMK, and $42 \mu \mathrm{M}$ and $74 \mu \mathrm{M}$ against $\mathrm{AKT}$, respectively), confirming their specificity towards PAK1.

\subsection{Anti-PAK1 activity of frondoside A (FRA)}

Extracts of several distinct sea cucumbers have been
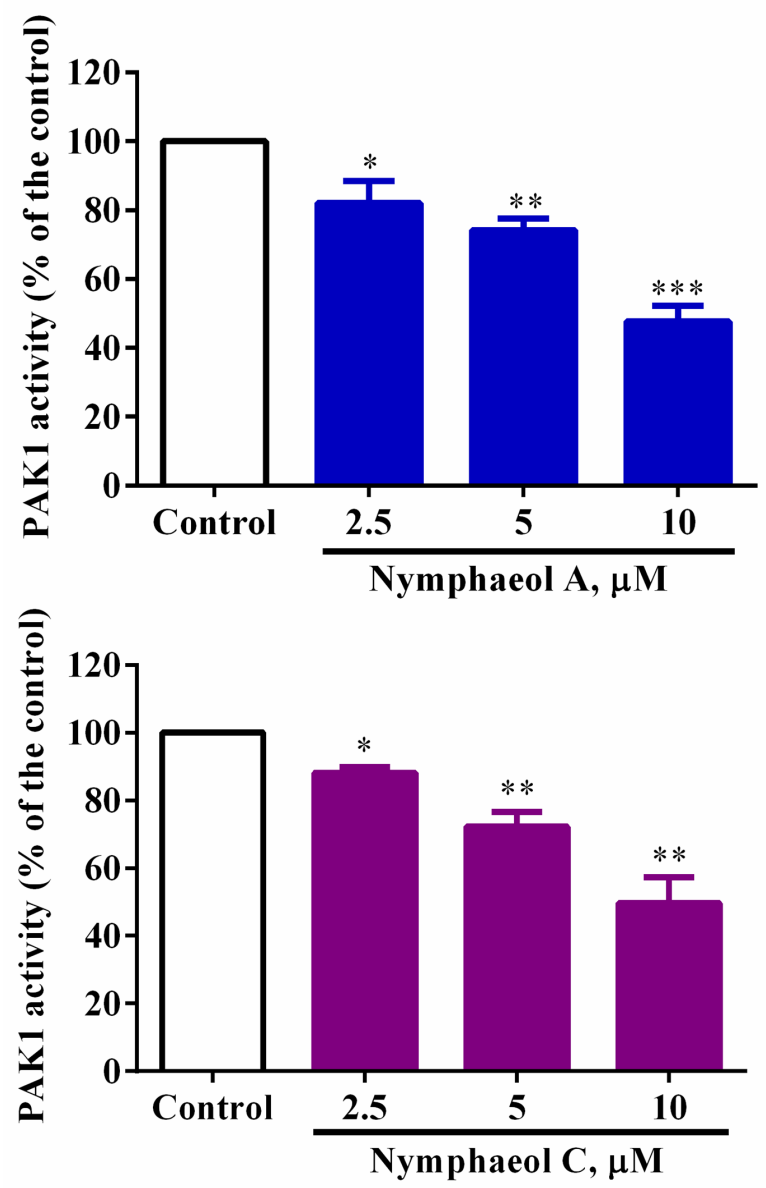

Figure 4. Nymphaeols directly inhibit PAK1 in vitro. PAK1 was treated in vitro with either nymphaeol A (left) or C (right) at the indicated concentrations. $\mathrm{IC}_{50}$ of both nymphaeols $\mathrm{A}$ and $\mathrm{C}$ is around $10 \mu \mathrm{M}$. Asterisks on each bar indicate significant differences between treatment and control. ${ }^{*} 0.01 \leq p \leq 0.05$, ** $p \leq 0.01,{ }^{* * *} p \leq 0.001$. shown to suppress the growth of cancer cells including A549 lung cancer and pancreatic cancer cell lines which carry the oncogenic Ki-RAS mutant $(8,9)$. In most cases, anti-cancer ingredients derived from sea cucumbers belong to sulfated saponins. Among these saponins, frondoside A (FRA) from Cucumaria frondosa is the most potent so far, inhibiting the PAK1dependent growth of A549 cancer cells with $\mathrm{IC}_{50}$ around $2.5 \mu \mathrm{M}$ for $24 \mathrm{~h}(9)$, but under our own culture conditions $(72 \mathrm{~h})$, the $\mathrm{IC}_{50}$ against $\mathrm{A} 549$ is around 0.6 $\mu \mathrm{M}$ (see Table 1). However, the precise molecular mechanism underlying its anti-cancer action still remains to be clarified.

A few previous observations raised the possibility that FRA might inhibit PAK1 directly (or indirectly): (i) FRA inhibits the PAK1-dependent growth of A549 cancer cells, (ii) FRA up-regulates p21 (CDK inhibitor) whose expression is suppressed by PAK1 (8-10), and (iii) nymphaeols from OP directly inhibits PAK1 in a selective manner (see Figure 4 and Table 1). Thus, we were prompted to test in vitro if FRA could inhibit PAK1 and a few other kinases as well. As shown in Figure 5, FRA directly inhibits PAK1 in vitro with $\mathrm{IC}_{50}$ around $1 \mu \mathrm{M}$, but both LIMK and AKT with $\mathrm{IC}_{50}$

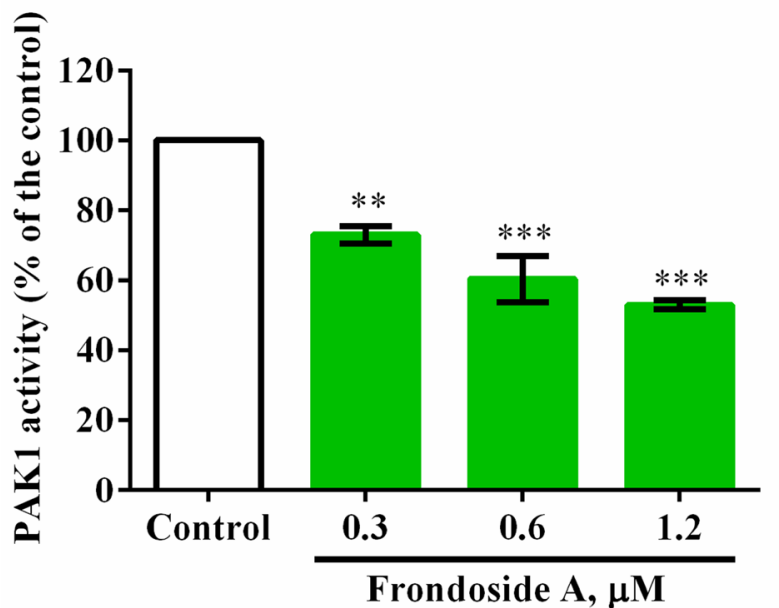

Figure 5. Frondoside A (FRA) directly inhibits PAK1 in vitro. PAK1 was treated in vitro with FRA at the indicated concentrations. The $\mathrm{IC}_{50}$ of FRA against PAK1 is around 1 $\mu \mathrm{M}$. Asterisks on each bar indicate significant differences between treatment and control. ${ }^{*} 0.01 \leq p \leq 0.05,{ }^{* *} p \leq 0.01$, $* * * p \leq 0.001$

Table 1. Anti-cancer activity and anti-kinase specificity of frondoside A and Okinawa propolis (OP) ingredients (nymphaeols)

\begin{tabular}{|c|c|c|c|c|}
\hline Items & Anti-cancer $\left(\mathrm{IC}_{50}\right)$ & Anti-PAK1 $\left(\mathrm{IC}_{50}\right)$ & Anti-LIMK $\left(\mathrm{IC}_{50}\right)$ & Anti-AKT $\left(\mathrm{IC}_{50}\right)$ \\
\hline Frondoside A & 0.6 & 1.2 & 60 & 59 \\
\hline Okinawa propolis & 12 & 10 & 39 & 30 \\
\hline Nymphaeol A & 3.6 & 9.6 & 161 & 42 \\
\hline Nymphaeol C & 6.5 & 9.8 & 171 & 74 \\
\hline Curcumin & 23 & 16 & 30 & 44 \\
\hline
\end{tabular}

$\mathrm{IC}_{50}$ value is in $\mu \mathrm{M}$, except for Okinawa propolis $(\mathrm{OP})$ in $\mu \mathrm{g} / \mathrm{mL}$. 
around $60 \mu \mathrm{M}$ (see Table 1), clearly indicating that FRA is indeed a potent PAK1-inhibitor, and its PAK1specificity is even far more profound than that of nymphaeols.

To the best of our knowledge, OP is the very first propolis that has been proven to directly inhibit PAK1. All other propolis products in market such as ARCbased GP and CAPE-based Bio 30 block PAK1 only indirectly (by down-regulating RAC or other activators of PAK1).

Back in 2003, we found a rare potent marine poison called ST-2001, a 3-OH derivative of Staurosporine (ST), which directly inhibits PAK1, PKC and several other kinases with $\mathrm{IC}_{50}$ around $1 \mathrm{nM}$ (13), but its antikinase mode of action is clearly "non-specific". Thus, to the best of our knowledge, FRA is the very first PAK1specific inhibitor of marine origin. Currently, we are testing if FRA also could extend the healthy lifespan of C. elegans, as does OP (7).

Regarding the structure-function relationship of nymphaeols (see Figure 1 for chemical structures), either the position of geranyl side chain in nymphaeols or an extra short side chain in nymphaeol $\mathrm{C}$ does not seem to affect either their anti-PAK1 activity or kinasespecificity. In an attempt to determine the specific role of geranyl side chains in either anti-cancer/cellpermeability or anti-PAK1 activity/kinase specificity if any, we are planning to study the potential anti-cancer and anti-PAK1 activity of far simpler flavonoids such as naringenin and sakuranetin which contain no geranyl side chain.

\section{Acknowledgements}

We are very grateful to Mr. Md Shahinozzaman for his technical assistance to confirm the $\mathrm{IC}_{50}$ of Frondoside A against A549 cancer cell growth. This study was supported in part by the fishing company "Jinsho", Japan.

\section{References}

1. Demestre M, Messerli SM, Celli N, Shahhossini M, Kluwe L, Mautner V, Maruta H. CAPE (caffeic acid phenethyl ester)-based propolis extract (Bio 30) suppresses the growth of human neurofibromatosis (NF) tumor xenografts in mice. Phytother Res. 2009; 23:226230.

2. Messerli SM, Ahn MR, Kunimasa K, Yanagihara M, Tatefuji T, Hashimoto K, Mautner V, Uto Y, Hori H, Kumazawa S, Kaji K, Ohta T, Maruta H. Artepillin C
(ARC) in Brazilian green propolis selectively blocks oncogenic PAK1 signaling and suppresses the growth of NF tumors in mice. Phytother Res. 2009; 23:423-427.

3. Maruta H. Herbal therapeutics that block the oncogenic kinase PAK1: A practical approach towards PAK1dependent diseases and longevity. Phytother Res. 2014; 28:656-672.

4. Tsuchiya I, Hosoya T, Ushida M, Kunimasa K, Ohta T, Kumazawa S. Nymphaeol-A isolated from Okinawan propolis suppresses angiogenesis and induces caspasedependent apoptosis via inactivation of survival signals. Evid Based Complement Alternat Med. 2013; 2013:826245.

5. Kumazawa S, Murase M, Momose N, Fukumoto S. Analysis of antioxidant prenylflavonoids in different parts of Macaranga tanarius, the plant origin of Okinawan propolis. Asian Pac J Trop Med. 2014; 7:16-20.

6. Inui S, Hosoya T, Kumazawa S. Hawaiian propolis: comparative analysis and botanical origin. Nat Prod Commun. 2014; 9:165-166.

7. Taira N, Nguyen BCQ, Be Tu PT, Tawata S. Effect of Okinawa Propolis on PAK1 activity, Caenorhabditis elegans longevity, melanogenesis, and growth of cancer cells. J Agric Food Chem. 2016; 64:5484-5489.

8. Li X, Roginsky AB, Ding XZ, Woodward C, Collin P, Newman RA, Bell RH Jr, Adrian TE. Review of the apoptosis pathways in pancreatic cancer and the antiapoptotic effects of the novel sea cucumber compound, Frondoside A. Ann N Y Acad Sci. 2008; 1138:181-198.

9. Attoub S, Arafat K, Gélaude A, Al Sultan MA, Bracke M, Collin P, Takahashi T, Adrian TE, De Wever O. Frondoside a suppressive effects on lung cancer survival, tumor growth, angiogenesis, invasion, and metastasis. PLoS One. 2013; 8:e53087.

10. Nheu T, He H, Hirokawa Y,Walker F, Wood J, Maruta H. PAK is essential for RAS-induced upregulation of cyclin D1 during the G1 to S transition. Cell Cycle. 2004; 3:7174.

11. Nguyen BCQ, Be Tu PT, Tawata S, Maruta H. Combination of immunoprecipitation (IP)-ATP_Glo kinase assay and melanogenesis for the assessment of potent and safe PAK1-blockers in cell culture. Drug Discov Ther. 2015; 9:289-295.

12. Mezna M, Wong AC, Ainger M, Scott RW, Hammonds T, Olson MF. Development of a high-throughput screening method for LIM kinase 1 using a luciferase-based assay of ATP consumption. J Biomol Screen. 2012; 17:460468.

13. Nheu TV, He H, Hirokawa Y, Tamaki K, Florin L, Schmitz ML, Suzuki-Takahashi I, Jorissen RN, Burgess AW, Nishimura S, Wood J, Maruta H. The K252a derivatives, inhibitors for the PAK/MLK kinase family selectively block the growth of RAS transformants. Cancer J. 2002; 8:328-336.

(Received February 13, 2017; Revised April 6, 2017; Accepted April 9, 2017) 